\title{
Outcomes of reoperative aortic valve replacement after previous sternotomy
}

Damien J. LaPar, MD, Zequan Yang, MD, George J. Stukenborg, PhD, Benjamin B. Peeler, MD, John A. Kern, MD, Irving L. Kron, MD, and Gorav Ailawadi, MD

Objective: Increasingly, patients with previous sternotomy require aortic valve replacement. We compared outcomes of reoperative aortic valve replacement after previous sternotomy and primary aortic valve replacement by surgical era. Effect of initial cardiac operation on reoperative aortic valve replacement was also investigated.

Methods: Between January 1996 and December 2007, a total of 1603 patients undergoing elective aortic valve replacement were entered prospectively into our clinical database. Patients were divided into eras A (1996-1999), B (2000-2003), and C (2004-2007). A total of 191 patients (12\%) had previous sternotomy for coronary artery bypass grafting $(n=88)$, coronary artery bypass grafting with aortic valve replacement $(n=16)$, aortic valve replacement with or without other aortic procedure $(n=70)$, and other cardiac procedures $(n=17)$. Mean ages were $66.5 \pm 13.1$ years in reoperative group and $65.5 \pm 14.9$ years in primary group.

Results: Mortality in reoperative group decreased significantly with time (A $15.4 \%$ vs B $15.1 \%$ vs C $2.0 \%, P=$ $.004)$ and was equivalent to primary group in era $\mathrm{C}(3.5 \%$ vs $2.0 \%, P=.65)$. Major complications also significantly decreased with time in reoperative group (A $25.6 \%$ vs B $17.0 \%$ vs C $6.1 \%, P=.006$ ). Importantly, patients had more comorbidities with time and increased preoperative risk in era $\mathrm{C}$. There were no differences in outcome by initial cardiac operation in reoperative group.

Conclusions: Reoperative aortic valve replacement now carries similar morbidity and mortality to primary replacement. Risk of reoperation is not affected by primary operation. (J Thorac Cardiovasc Surg 2010;139:263-72)

Aortic valve replacement (AVR) is a frequently performed operation in the United States. According to the Society of Thoracic Surgeons (STS) national database, approximately 12,000 to 18,000 cases of isolated AVR and a similar number of AVR procedures with concomitant coronary artery bypass grafting $(\mathrm{CABG})$ are performed annually. ${ }^{1}$ The operative mortalities for isolated AVR now approach $3 \%$, whereas the mortality for AVR with CABG is approximately $5 \% .{ }^{1}$ With a rising elderly population and improvements in surgical management of disease, patients are living longer after cardiac surgery and facing increasing preoperative risks. Consequently, a higher percentage of patients with aortic valve disease are likely to undergo reoperative AVR in the foreseeable future. Furthermore, advancing technology with the use of percutaneous valves has recently emerged for the treatment of aortic valve disease in high-risk patients.

In the past, reoperative AVR was considered a high-risk operation. Previous studies have demonstrated mortalities

\footnotetext{
From the Department of Surgery, University of Virginia, Charlottesville, Va. Disclosures: None.

Read at the Eighty-ninth Annual Meeting of The American Association for Thoracic Surgery, Boston, Mass, May 9-13, 2009.

Received for publication April 30, 2009; revisions received July 17, 2009; accepted for publication Sept 4, 2009; available ahead of print Dec 14, 2009.

Address for reprints: Gorav Ailawadi, MD, PO Box 800679, Charlottesville, VA 22908-0679 (E-mail: gorav@virginia.edu).

$0022-5223 / \$ 36.00$

Copyright $(c) 2010$ by The American Association for Thoracic Surgery doi:10.1016/j.jtcvs.2009.09.006
}

for reoperative AVR to range from $5.9 \%$ to $14 \% .^{2-4}$ Recent trends, however, have demonstrated improved outcomes for reoperative AVR. ${ }^{5,6}$ Accordingly, we compared operative outcomes of reoperative AVR with primary AVR by surgical era. We hypothesized that reoperative AVR would demonstrate similar risks to primary AVR with respect to operative mortality and major complications.

\section{MATERIALS AND METHODS \\ Patients}

This investigation was approved by the human investigation committee of the University of Virginia Health System (HSR 14077), including a waiver of the need to obtain patient consent. Data from all patients undergoing aortic valve operations at our institution were entered prospectively into the STS database. A retrospective review was then performed of all patients undergoing AVR from January 1996 to December 2007. Primary AVR occurred in the absence of a previous sternotomy or cardiac operation while reoperative AVRs followed a previous sternotomy for cardiac operation. We stratified patients according to extent of cardiac operation to further delineate any effects of concomitant operations. Further, to evaluate the impact of the initial operation, patients undergoing reoperative AVR were categorized into 4 groups according to previous cardiac operation: (1) CABG only, (2) CABG with AVR, (3) AVR with or without other aortic operation, and (4) other cardiac procedures. In addition, reoperative AVRs were divided into 3 operative eras (A, 1996-1999; B, 2000-2003; C, 2004 2007) to assess the impact of operative era on AVR.

Patient demographic characteristics, preoperative risk factors, operative features, and postoperative outcomes were examined over time. STS definitions were used to describe all preoperative variables, postoperative complications, and outcomes. Standard and logistic EuroSCOREs were calculated to evaluate preoperative risk. The method used to calculate the STS predicted risk of mortality has undergone multiple changes and revisions 


\section{Abbreviations and Acronyms \\ $\mathrm{AVR}=$ aortic valve replacement \\ $\mathrm{CABG}=$ coronary artery bypass grafting \\ STS $=$ Society of Thoracic Surgeons}

during our study period. Moreover, the STS model is unable to provide a risk score for many patients undergoing concomitant operations. We therefore chose not to use that risk assessment model in our data analyses. Operative mortality included patient deaths occurring before hospital discharge or within 30 days after the operation. Major complications included the composite incidence of postoperative renal failure, stroke, and pneumonia.

\section{Statistical Analysis}

The primary outcomes measured were operative mortality and major complication rate for patients undergoing primary and reoperative AVR. Patients undergoing reoperative AVR were further analyzed with respect to operative era as well as initial operation. All patient group comparisons were unpaired. Categoric variables were analyzed by bivariate comparisons with either $\chi^{2}$ test or Fisher's Exact Test as indicated. Analysis of variance was used for all continuous variables.

Multivariable logistic regression was performed to estimate the odds of death associated with reoperative AVR. All preoperative variables entered as covariates (male sex, peripheral vascular disease, cerebrovascular disease, endocarditis, chronic renal insufficiency, chronic lung disease, coronary artery disease, and operative era) were selected a priori according to established clinical risk in cardiac operations. The estimated odds of death were adjusted for all covariates. The discrimination achieved by these models was assessed with the $\mathrm{C}$ statistic, which is equivalent to the area under the receiver operating characteristic curve. $C$ statistic values of 1.0 indicate perfect discrimination between survivors and decedents, whereas values of 0.5 indicate results equal to chance.

All categoric variables are expressed as percentage of group of origin, and continuous variables are expressed as mean $\pm \mathrm{SD}$. Odds ratios with $95 \%$ confidence intervals are used to report the results of the logistic regression. All $P$ values reported are 2 -tailed. Data manipulation and analysis were performed with SAS version 9.1.3 software (SAS Institute, Inc, Cary, NC).

\section{RESULTS}

\section{Comparison of Primary and Reoperative AVR}

During an 11-year study period, a total of 1603 patients underwent AVR at the University of Virginia. Within this cohort, 1412 primary AVRs and 191 reoperative AVRs were performed (Table 1). The average patient age was similar between primary and reoperative AVR groups. The reoperative AVR group contained more male patients than did the primary AVR group and had higher rates of preoperative peripheral vascular disease, diabetes, dyslipidemia, coronary artery disease, endocarditis, and chronic renal insufficiency. Conversely, patients undergoing primary AVR were in New York Heart Association functional class IV more commonly than were patients undergoing reoperative AVR.

Operative features also differed between primary and reoperative AVR groups (Table 1). Patients in the primary AVR group underwent significantly more isolated AVR operations than did those in the reoperative AVR group.
Patients undergoing reoperative AVR had higher rates of concomitant $\mathrm{CABG}$ and mitral valve procedures than did those undergoing primary AVR. The primary AVR group underwent more aortic root replacements and pulmonic valve procedures than did the reoperative AVR group. The reoperative AVR group also underwent placement of more early mechanical valves than did the primary AVR group. Aortic crossclamp times were shorter in reoperative AVRs than in primary AVRs, whereas cardiopulmonary bypass times were similar between groups.

Few differences in postoperative outcomes were observed between groups. Overall major complication rates were equivalent between primary and reoperative AVR groups $(15.2 \%$ [214/1412] vs $15.2 \%$ [29/191], respectively, $P=$ .92). Patients undergoing primary AVR had significantly higher rates of sepsis $(2.9 \%$ [41/1412] vs $0.0 \%$ [0/191], $P=.01)$, atrial fibrillation $(23.9 \%[337 / 1412]$ vs $13.6 \%$ [26/191], $P=.001)$, and prolonged ventilation $(10.0 \%$ $[141 / 1,412]$ vs $3.7 \%[7 / 191], P=.003)$ than did those undergoing reoperative AVR. Total hospital stay $(9.0 \pm 9.6$ days vs $9.1 \pm 9.7$ days, $P=.89)$ and intensive care unit stay ( $3.3 \pm 7.3$ days vs $4.1 \pm 8.5$ days, $P=.15$ ) were similar between primary and reoperative AVR groups. In an analysis of isolated AVR operations, higher rates of major complications $(16.0 \%$ [181/1131] vs $8.7 \%$ [9/104], $P=.04)$, atrial fibrillation $(24.6 \%$ [278/1131] vs $15.4 \%$ [16/104], $P=$ $.04)$, and prolonged ventilation $(10.9 \%$ [123/1131] vs $2.9 \%$ [3/104], $P=.01$ ) were observed among patients undergoing primary AVR than among patients undergoing reoperative AVR.

The overall operative mortality for reoperative AVR was higher than for primary AVR (8.4\% [16/191] vs 4.1\% [58/ 1412 ], $P=.02$ ). Among the subgroup of patients undergoing isolated AVR, higher overall operative mortality was also observed for reoperative AVR than for primary AVR $(8.7 \%$ [9/104] vs. $4.1 \%$ [46/1131], $P=.04)$.

\section{Influence of Initial Operation on Reoperative AVR}

An analysis of all reoperative AVR cases $(n=191)$ stratified by initial cardiac operation revealed that 88 patients $(46.1 \%)$ had undergone previous CABG only, 16 patients (8.4\%) had undergone previous CABG and AVR, 70 patients $(36.6 \%)$ had undergone previous AVR with or without other aortic procedures, and 17 patients $(8.9 \%)$ had undergone other previous cardiac procedures. Postoperative outcomes were similar among these reoperative AVR groups despite differences in initial cardiac operation. Specifically, major complication rates were not statistically different after initial CABG only (14.8\% [13/88]), CABG and AVR (25.0\% [4/16]), AVR with or without other aortic procedures $(8.6 \%[6 / 70])$, and other cardiac procedures $(23.5 \%$ [4/17], $P=.80$ ). Operative mortality also did not differ statistically among initial operation groups: CABG only $5.7 \%$ (5/88), CABG and AVR 18.8\% (3/16), AVR with or 
TABLE 1. Preoperative risk factors and operative features for patients undergoing primary versus reoperative aortic valve replacement $(\mathbf{n}=1603)$

\begin{tabular}{|c|c|c|c|}
\hline Variable & Primary $(n=1412)$ & Reoperative $(\mathbf{n}=191)$ & $P$ value \\
\hline \multicolumn{4}{|l|}{ Preoperative } \\
\hline Age at operation $(y$, mean $\pm S D)$ & $65.5 \pm 14.9$ & $66.5 \pm 13.1$ & .41 \\
\hline Male sex (no.) & $862(61.0 \%)$ & $140(73.3 \%)$ & .001 \\
\hline Peripheral vascular disease (no.) & $123(8.7 \%)$ & $33(17.3 \%)$ & .001 \\
\hline Cerebrovascular disease (no.) & $317(22.4 \%)$ & $37(19.4 \%)$ & .35 \\
\hline Chronic lung disease (no.) & $191(13.5 \%)$ & $27(14.1 \%)$ & .82 \\
\hline Diabetes (no.) & $258(18.3 \%)$ & $50(26.2 \%)$ & .01 \\
\hline Dyslipidemia (no.) & $597(42.3 \%)$ & $108(56.5 \%)$ & $<.001$ \\
\hline Coronary artery disease (no.) & $475(33.6 \%)$ & $120(62.8 \%)$ & $<.001$ \\
\hline Hypertension (no.) & $827(58.6 \%)$ & $111(58.1 \%)$ & .94 \\
\hline \multicolumn{4}{|l|}{ New York Heart Association functional class (no.) } \\
\hline I & $597(42.3 \%)$ & $14(7.3 \%)$ & $<.001$ \\
\hline II & $360(25.5 \%)$ & $104(54.5 \%)$ & $<.001$ \\
\hline III & $298(21.1 \%)$ & $63(33.0 \%)$ & $<.001$ \\
\hline IV & $156(11.0 \%)$ & $10(5.2 \%)$ & .01 \\
\hline Endocarditis (no.) & $61(4.3 \%)$ & $15(7.9 \%)$ & .04 \\
\hline Chronic renal insufficiency (no.) & $154(10.9 \%)$ & $32(16.8 \%)$ & .02 \\
\hline \multicolumn{4}{|l|}{ Operative } \\
\hline Isolated aortic valve replacement (no.) & $1,131(80.1 \%)$ & $104(54.5 \%)$ & $<.001$ \\
\hline Bioprosthetic valve (no.) & $939(66.5 \%)$ & $125(65.5 \%)$ & .81 \\
\hline Mechanical valve (no.) & $299(21.2 \%)$ & $55(28.8 \%)$ & .02 \\
\hline Homograft (no.) & $35(2.5 \%)$ & $5(2.6 \%)$ & .81 \\
\hline \multicolumn{4}{|l|}{ Concomitant operations (no.) } \\
\hline Coronary artery bypass grafting & $126(8.9 \%)$ & $58(30.4 \%)$ & $<.001$ \\
\hline Aortic root operations & $132(9.3 \%)$ & $9(4.7 \%)$ & .04 \\
\hline Mitral valve procedures & $69(4.9 \%)$ & $22(11.5 \%)$ & $<.001$ \\
\hline Pulmonic valve procedures & $37(2.6 \%)$ & $0(0.0 \%)$ & .02 \\
\hline Tricuspid valve procedures & $23(1.6 \%)$ & $5(2.6 \%)$ & .37 \\
\hline Cardiopulmonary bypass time (min, mean $\pm \mathrm{SD})$ & $137.9 \pm 58.8$ & $135.4 \pm 46.7$ & .66 \\
\hline Aortic crossclamp time (min, mean \pm SD) & $101.5 \pm 44.2$ & $92.2 \pm 38.4$ & .03 \\
\hline
\end{tabular}

without other aortic procedures $8.6 \%(6 / 70)$, and other cardiac procedures $11.8 \%(2 / 17, P=.54)$.

\section{Outcomes of Reoperative AVR by Operative Era}

Reoperative AVRs were analyzed according to operative era (Table 2). Significantly more reoperative AVRs were performed in the most recent era. Compared with early operative eras, patients in the most recent era were older and had higher preoperative EuroSCOREs. Higher rates of preoperative dyslipidemia, coronary artery disease, and hypertension were also observed in the most recent era. Alternatively, New York Heart Association functional class III was more common in the earliest operative era than in the most recent operative era (era A $48.7 \%$ vs era C $31.3 \%$, $P<.001)$. The duration between the initial operation and the reoperative AVR was longest in the most recent era. Other preoperative risk factors, including endocarditis, were similar over time. Intraoperatively, higher rates of bioprosthetic valves were used in era $\mathrm{C}$, whereas mechanical valves were more frequently used in early eras. Moreover, both cardiopulmonary bypass time (era A $157.4 \pm 47.5$ minutes vs era $\mathrm{C} 132.7 \pm 44.8$ minutes, $P=.005$ ) and aortic crossclamp time (era A $108.6 \pm 37.2$ minutes vs era C $89.4 \pm 36.4$ minutes, $P=.006$ ) decreased significantly over time.

Operative outcomes for reoperative AVR improved over time (Table 3). Reoperations for postoperative bleeding or tamponade, postoperative pneumonia, and postoperative renal failure were more common in earlier operative eras than in the most recent era. Hospital and intensive care unit stays were similar across eras. Major complication rate decreased significantly over time. Operative mortality also decreased over time; in the most recent era, mortality for reoperative AVR was similar to that for primary AVR $(2.0 \%$ [2/99] vs $3.5 \%$ [19/542], $P=.65$; Figure 1 ).

\section{Isolated Reoperative AVR by Operative Era}

A total of 104 patients $(54.5 \%)$ underwent isolated reoperative AVR (Table 4). Time-related trends observed among these patients were nearly identical to those among all reoperative AVR patients. In the most recent operative era, patients were older and had higher rates of dyslipidemia, coronary artery disease, and hypertension. Additionally, patients in the most recent era had higher 
TABLE 2. Preoperative risk factors and operative features for patients undergoing reoperative aortic valve replacement with respect to operative era $(\mathbf{n}=191)$

\begin{tabular}{|c|c|c|c|c|}
\hline Variable & $1996-1999(n=39)$ & $2000-2003(n=53)$ & 2004-2007 $(n=99)$ & $P$ value \\
\hline \multicolumn{5}{|l|}{ Preoperative } \\
\hline Age at operation $(y$, mean $\pm S D)$ & $64.7 \pm 12.1$ & $63.6 \pm 17.4$ & $68.7 \pm 10.3$ & 0.04 \\
\hline Male sex (no.) & $25(64.1 \%)$ & $42(79.2 \%)$ & $73(73.7 \%)$ & 0.27 \\
\hline Peripheral vascular disease (no.) & $3(7.7 \%)$ & $7(13.2 \%)$ & $23(23.2 \%)$ & 0.06 \\
\hline Cerebrovascular disease (no.) & $4(10.3 \%)$ & $10(18.9 \%)$ & $23(23.2 \%)$ & 0.22 \\
\hline Chronic lung disease (no.) & $10(25.6 \%)$ & $7(13.2 \%)$ & $10(10.1 \%)$ & 0.06 \\
\hline Diabetes (no.) & $11(28.2 \%)$ & $9(17.0 \%)$ & $30(30.3 \%)$ & 0.20 \\
\hline Dyslipidemia (no.) & $10(25.6 \%)$ & $22(41.5 \%)$ & $76(76.8 \%)$ & $<.001$ \\
\hline Coronary artery disease (no.) & $12(30.8 \%)$ & $26(49.1 \%)$ & $82(82.8 \%)$ & $<.001$ \\
\hline Hypertension (no.) & $15(38.5 \%)$ & $28(52.8 \%)$ & $68(68.7 \%)$ & .003 \\
\hline \multicolumn{5}{|l|}{ New York Heart Association functional class (no.) } \\
\hline I & $7(17.9 \%)$ & $5(9.4 \%)$ & $2(2.0 \%)$ & .004 \\
\hline II & $10(25.6 \%)$ & $30(56.6 \%)$ & $64(64.6 \%)$ & $<.001$ \\
\hline III & $19(48.7 \%)$ & $13(24.5 \%)$ & $31(31.3 \%)$ & .04 \\
\hline IV & $3(7.7 \%)$ & $5(9.4 \%)$ & $2(2.0 \%)$ & .11 \\
\hline Endocarditis (no.) & $4(10.3 \%)$ & $5(9.4 \%)$ & $6(6.1 \%)$ & .63 \\
\hline Chronic renal insufficiency (no.) & $3(7.7 \%)$ & $7(13.2 \%)$ & $22(22.2 \%)$ & .09 \\
\hline Ejection fraction $(\%$, mean $\pm \mathrm{SD})$ & $44.0 \pm 14.9$ & $57.7 \pm 14.9$ & $47.4 \pm 14.3$ & $<.001$ \\
\hline EuroSCORE (logistic, mean \pm SD) & $19.6 \pm 1.8$ & $18.8 \pm 1.6$ & $25.4 \pm 1.9$ & $<.001$ \\
\hline EuroSCORE (standard, mean $\pm \mathrm{SD}$ ) & $9.4 \pm 0.4$ & $9.5 \pm 0.3$ & $10.8 \pm 0.3$ & $<.001$ \\
\hline Time to reoperation $(\mathrm{y}$, mean $\pm \mathrm{SD})$ & $9.6 \pm 0.8$ & $11.5 \pm 1.0$ & $12.1 \pm 0.9$ & $<.001$ \\
\hline \multicolumn{5}{|l|}{ Operative } \\
\hline Isolated aortic valve replacement (no.) & $19(48.7 \%)$ & $30(56.6 \%)$ & $55(55.6 \%)$ & .72 \\
\hline Bioprosthetic valve (no.) & $16(41.0 \%)$ & $34(64.2 \%)$ & $75(75.8 \%)$ & .001 \\
\hline Mechanical valve (no.) & $19(48.7 \%)$ & $14(26.4 \%)$ & $22(22.2 \%)$ & .01 \\
\hline Homograft (no.) & $1(2.6 \%)$ & $3(5.7 \%)$ & $1(1.0 \%)$ & .23 \\
\hline \multicolumn{5}{|l|}{ Concomitant operations (no.) } \\
\hline Coronary artery bypass grafting & $15(38.5 \%)$ & $12(22.6 \%)$ & $31(31.3 \%)$ & .25 \\
\hline Aortic root operations & $0(0.0 \%)$ & $3(5.7 \%)$ & $6(6.1 \%)$ & .30 \\
\hline Mitral valve procedures & $6(15.4 \%)$ & $9(17.0 \%)$ & $7(7.1 \%)$ & .13 \\
\hline Pulmonic valve procedures & $0(0.0 \%)$ & $0(0.0 \%)$ & $0(0.0 \%)$ & NA \\
\hline Tricuspid valve procedures & $0(0.0 \%)$ & $0(0.0 \%)$ & $4(4.0 \%)$ & .15 \\
\hline Cardiopulmonary bypass time (min, mean $\pm \mathrm{SD}$ ) & $157.4 \pm 47.5$ & $131.6 \pm 58.7$ & $132.7 \pm 44.8$ & .18 \\
\hline Aortic crossclamp time (min, mean \pm SD) & $108.6 \pm 37.2$ & $95.1 \pm 53.6$ & $89.4 \pm 36.4$ & .21 \\
\hline
\end{tabular}

NA, Not applicable.

EuroSCOREs than did those in the early eras. Cardiopulmonary bypass time (era A $137.2 \pm 37.9$ minutes vs era C $115.7 \pm 37.5$ minutes, $P=.04)$ and aortic crossclamp time (era A $91.7 \pm 20.9$ minutes vs era $\mathrm{C} 73.8 \pm 31.5$ minutes, $P=.02$ ) were significantly shorter in the most recent era. Decreased rates of postoperative pneumonia, prolonged ventilation, and renal failure were noted over time among patients undergoing isolated reoperative AVR. Operative mortality also decreased significantly with time for isolated reoperative AVR and within the most recent era was equivalent to that of isolated primary AVR $(0.0 \%[0 / 55]$ vs $3.3 \%$ [16/486], $P=.39$ ).

\section{Factors Related to Mortality in Reoperative AVR}

A total of $16(8.4 \%)$ patients in the reoperative AVR group died: 7 patients $(43.8 \%)$ of postoperative bleeding or tamponade, 3 patients $(18.8 \%)$ of multiorgan failure,
2 patients $(12.5 \%)$ of cardiac arrest, 2 patients $(12.5 \%)$ of heart failure, 1 patient $(6.3 \%)$ of respiratory failure, and 1 patient $(6.3 \%)$ of stroke. All 6 deaths in era A $(100 \%)$ occurred after elective repeat AVR, whereas $75 \%$ of deaths in era B (6/8) occurred after urgent AVR and $25 \%(2 / 8)$ after emergency AVR. Within era C, all deaths $(100 \%$ [2/2]) occurred after urgent AVR. Significantly more deaths occurred after urgent, reoperative AVR in the recent eras (era A $0.0 \%$ vs era $\mathrm{B} 75.0 \%$ vs era $\mathrm{C} 100.0 \%, P=.01)$.

Univariate analyses (Table 5) of all risk factors related to mortality in reoperative AVR revealed that coronary artery disease and hypertension were paradoxically more frequent among patients who had survived than among those who died. Higher preoperative EuroSCOREs were noted for patients who died. All other risk factors were similar between groups. Patients who died had longer cardiopulmonary 
TABLE 3. Postoperative outcomes for patients undergoing reoperative aortic valve replacement with respect to operative era $(\mathrm{n}=191)$

\begin{tabular}{|c|c|c|c|c|}
\hline Variable & 1996-1999 $(n=39)$ & $2000-2003(n=53)$ & 2004-2007 $(n=99)$ & $P$ value \\
\hline Sepsis (no.) & $0(0.0 \%)$ & $0(0.0 \%)$ & $0(0.0 \%)$ & NA \\
\hline Stroke (no.) & $2(5.1 \%)$ & $4(7.5 \%)$ & $4(4.0 \%)$ & .65 \\
\hline Cardiac arrest (no.) & $1(2.6 \%)$ & $1(1.9 \%)$ & $1(1.0 \%)$ & .79 \\
\hline Reoperation for bleeding or tamponade (no.) & $1(2.6 \%)$ & $8(15.1 \%)$ & $5(5.1 \%)$ & .03 \\
\hline Atrial fibrillation (no.) & $7(17.9 \%)$ & $7(13.2 \%)$ & $12(12.1 \%)$ & .66 \\
\hline Heart block (no.) & $0(0.0 \%)$ & $0(0.0 \%)$ & $3(3.0 \%)$ & .24 \\
\hline Gastrointestinal event (no.) & $1(2.6 \%)$ & $0(0.0 \%)$ & $1(1.0 \%)$ & .49 \\
\hline Pneumonia (no.) & $3(7.7 \%)$ & $5(9.4 \%)$ & $0(0.0 \%)$ & .01 \\
\hline Prolonged ventilation (no.) & $3(7.7 . \%)$ & $3(5.7 \%)$ & $1(1.0 \%)$ & .11 \\
\hline Renal failure (no.) & $5(12.8 \%)$ & $4(7.5 \%)$ & $2(2.0 \%)$ & .04 \\
\hline Hemodialysis (no.) & $1(2.6 \%)$ & $2(3.8 \%)$ & $0(0.0 \%)$ & .17 \\
\hline Hospital stay $(\mathrm{d}$, mean $\pm \mathrm{SD})$ & $10.6 \pm 8.3$ & $8.9 \pm 11.5$ & $8.7 \pm 9.2$ & .58 \\
\hline Intensive care unit stay $(\mathrm{d}$, mean $\pm \mathrm{SD})$ & $4.8 \pm 6.9$ & $4.3 \pm 9.8$ & $3.8 \pm 8.5$ & .83 \\
\hline Major complications (no.) & $10(25.6 \%)$ & $9(17.0 \%)$ & $6(6.1 \%)$ & .006 \\
\hline Operative mortality (no.) & $6(15.4 \%)$ & $8(15.1 \%)$ & $2(2.0 \%)$ & .004 \\
\hline
\end{tabular}

NA, Not applicable.

bypass and aortic crossclamp times than did survivors. All other operative features were similar between groups.

A multivariable logistic regression analysis examining the outcome of operative mortality among patients undergoing reoperative AVR identified early operative era as the only significant predictor of mortality (odds ratio $7.39,95 \%$ confidence interval $1.432-38.115, P=.02$ ). The model achieved adequate discrimination with a $\mathrm{C}$ statistic of 0.796 .

\section{DISCUSSION}

In this study, we corroborated current evidence suggesting recent declines in operative mortality and major complication rate associated with reoperative AVR. The reoperative AVR group had higher rates of preoperative comorbidities. Despite this elevated risk, the observed operative mortalities for both isolated reoperative AVR and reoperative AVR with concomitant operations significantly decreased with time and in the most recent era were comparable to those of primary AVR. Among patients undergoing reoperative AVR, we were unable to identify any significant differences related to the initial cardiac operation. Moreover, we identified early operative era as a significant predictor of mortality for patients undergoing reoperative AVR. We believe these results have the potential to change the status of reoperative AVR surgery.

Many studies have identified risk factors during reoperative AVR. Cardiac surgery in the elderly population adds risk to the patient. ${ }^{7,8}$ Other operative risk factors that have been identified include female sex, weight, cardiac functional class, endocarditis, reduced ejection fraction, peripheral vascular disease, renal insufficiency, concomitant CABG, number of coronary artery bypasses, prosthetic valve type or thrombosis, other cardiac valve disease, hemodynamic instability, and ascending aortic aneurysm repair., ${ }^{2,9-13}$

Despite having more risk factors and undergoing more concomitant CABG or mitral valve procedures, our reoperative AVR group had shorter aortic crossclamp times than did our primary AVR group. The longer aortic crossclamp times in primary AVRs may result from the higher percentage of aortic root operations performed in this population. Otherwise, crossclamp times among reoperative AVRs

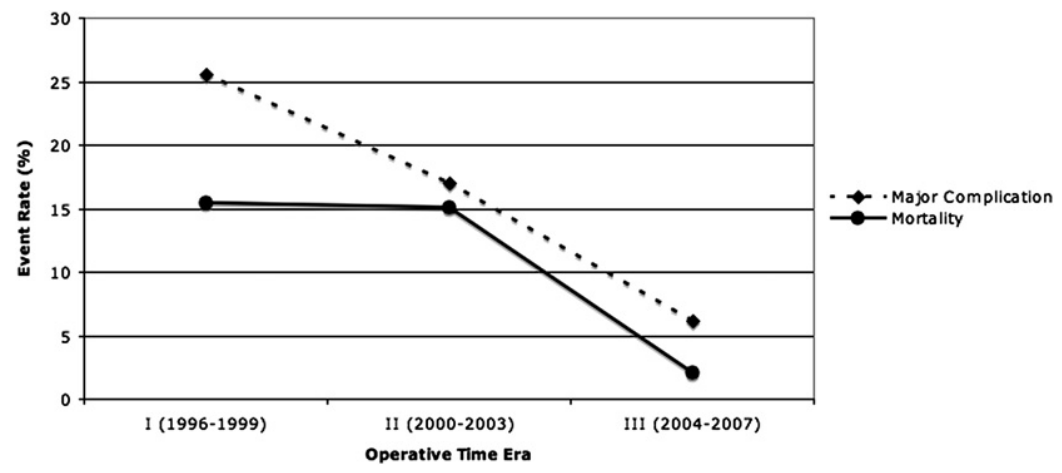

FIGURE 1. Mortality and major cofmplication rate for patients undergoing aortic valve replacement as function of operative era. 
TABLE 4. Preoperative risk factors, operative features, and postoperative outcomes for patients undergoing isolated reoperative aortic valve replacement with respect to operative era $(n=104)$

\begin{tabular}{|c|c|c|c|c|}
\hline Variable & $1996-1999(n=19)$ & $2000-2003(n=30)$ & 2004-2007 $(n=55)$ & $P$ value \\
\hline \multicolumn{5}{|l|}{ Preoperative } \\
\hline Age at operation $(y$, mean $\pm \mathrm{SD})$ & $59.3 \pm 14.8$ & $62.5 \pm 20.6$ & $69.4 \pm 11.1$ & .02 \\
\hline Male sex (no.) & $12(63.2 \%)$ & $23(76.7 \%)$ & $44(80.0 \%)$ & .33 \\
\hline Peripheral vascular disease (no.) & $3(15.8 \%)$ & $5(16.7 \%)$ & $15(27.3 \%)$ & .41 \\
\hline Cerebrovascular disease (no.) & $1(5.3 \%)$ & $6(20.0 \%)$ & $11(20.0 \%)$ & .31 \\
\hline Chronic lung disease (no.) & $6(31.6 \%)$ & $5(16.7 \%)$ & $6(10.9 \%)$ & .11 \\
\hline Diabetes (no.) & $4(21.1 \%)$ & $7(23.3 \%)$ & $17(30.9 \%)$ & .62 \\
\hline Dyslipidemia (no.) & $3(15.8 \%)$ & $14(46.7 \%)$ & $44(80.0 \%)$ & $<.001$ \\
\hline Coronary artery disease (no.) & $7(36.8 \%)$ & $17(56.7 \%)$ & $43(78.2 \%)$ & .003 \\
\hline Hypertension (no.) & $6(31.6 \%)$ & $16(53.3 \%)$ & $42(76.4 \%)$ & .001 \\
\hline \multicolumn{5}{|l|}{ New York Heart Association functional class (no.) } \\
\hline I & $5(26.3 \%)$ & $3(10.0 \%)$ & $1(1.8 \%)$ & .004 \\
\hline II & $3(15.8 \%)$ & $16(53.3 \%)$ & $34(61.8 \%)$ & .002 \\
\hline III & $10(52.6 \%)$ & $7(23.3 \%)$ & $19(34.5 \%)$ & .11 \\
\hline IV & $1(5.3 \%)$ & $4(13.3 \%)$ & $1(1.8 \%)$ & .09 \\
\hline Endocarditis (no.) & $3(15.8 \%)$ & $2(6.7 \%)$ & $4(7.3 \%)$ & .47 \\
\hline Chronic renal insufficiency (no.) & $1(5.3 \%)$ & $2(6.7 \%)$ & $10(18.2 \%)$ & .17 \\
\hline Ejection fraction $(\%$, mean $\pm \mathrm{SD})$ & $45 \pm 16.1$ & $53.9 \pm 13.2$ & $47.9 \pm 15.5$ & .09 \\
\hline EuroSCORE (logistic, mean \pm SD) & $20.1 \pm 8.4$ & $18.3 \pm 11.3$ & $27.5 \pm 18.2$ & .02 \\
\hline EuroSCORE (standard, mean $\pm \mathrm{SD}$ ) & $10.2 \pm 1.9$ & $9.6 \pm 2.2$ & $11.1 \pm 3.0$ & .04 \\
\hline \multicolumn{5}{|l|}{ Operative } \\
\hline Bioprosthetic valve (no.) & $5(26.3 \%)$ & $19(63.3 \%)$ & $45(81.8 \%)$ & $<.001$ \\
\hline Mechanical valve (no.) & $11(57.9 \%)$ & $6(20.0 \%)$ & $10(18.2 \%)$ & .002 \\
\hline Homograft (no.) & $0(0.0 \%)$ & $3(10.0 \%)$ & $0(0.0 \%)$ & NA \\
\hline Cardiopulmonary bypass time (min, mean $\pm \mathrm{SD}$ ) & $137.2 \pm 37.9$ & $95.5 \pm 28.6$ & $115.7 \pm 37.5$ & .16 \\
\hline Aortic crossclamp time (min, mean $\pm \mathrm{SD}$ ) & $91.7 \pm 20.9$ & $58.8 \pm 11.7$ & $73.8 \pm 31.5$ & .17 \\
\hline \multicolumn{5}{|l|}{ Outcome } \\
\hline Sepsis (no.) & $0(0.0 \%)$ & $0(0.0 \%)$ & $0(0.0 \%)$ & NA \\
\hline Stroke (no.) & $0(0.0 \%)$ & $2(6.7 \%)$ & $2(3.6 \%)$ & .50 \\
\hline Cardiac arrest (no.) & $0(0.0 \%)$ & $1(3.3 \%)$ & $1(1.8 \%)$ & .71 \\
\hline Reoperation for bleeding or tamponade (no.) & $0(0.0 \%)$ & $3(10.0 \%)$ & $1(1.8 \%)$ & .11 \\
\hline Atrial fibrillation (no.) & $4(21.1 \%)$ & $3(10.0 \%)$ & $9(16.4 \%)$ & .56 \\
\hline Heart block (no.) & $0(0.0 \%)$ & $0(0.0 \%)$ & $1(1.8 \%)$ & .64 \\
\hline Gastrointestinal event (no.) & $0(0.0 \%)$ & $0(0.0 \%)$ & $0(0.0 \%)$ & NA \\
\hline Pneumonia (no.) & $0(0.0 \%)$ & $5(16.7 \%)$ & $0(0.0 \%)$ & .002 \\
\hline Prolonged ventilation (no.) & $0(0.0 \%)$ & $3(10.0 \%)$ & $0(0.0 \%)$ & .02 \\
\hline Renal failure (no.) & $1(5.3 \%)$ & $2(6.7 \%)$ & $1(1.8 \%)$ & .51 \\
\hline Hemodialysis (no.) & $0(0.0 \%)$ & $2(6.7 \%)$ & $0(0.0 \%)$ & .08 \\
\hline Hospital stay (d, mean $\pm \mathrm{SD}$ ) & $8.4 \pm 4.9$ & $10.4 \pm 15.0$ & $7.6 \pm 4.2$ & .40 \\
\hline Intensive care unit stay $(\mathrm{d}$, mean $\pm \mathrm{SD})$ & $3.5 \pm 5.6$ & $5.9 \pm 12.6$ & $2.8 \pm 2.2$ & .18 \\
\hline Major complications (no.) & $1(5.3 \%)$ & $5(16.7 \%)$ & $3(5.5 \%)$ & .18 \\
\hline Operative mortality (no.) & $3(15.8 \%)$ & $6(20.0 \%)$ & $0(0.0 \%)$ & .003 \\
\hline
\end{tabular}

NA, Not applicable.

decreased over time. We believe that this trend reflects our improved operative technique and efficiency with time.

Recent series have documented improved operative mortality $(<5 \%)$ for reoperative AVR. ${ }^{5,6,14}$ These trends are consistent with our results; we report an overall operative mortality of $8.4 \%$ for reoperative AVR, which decreased to $2.0 \%$ in the most recent era. Not surprisingly, univariate analyses demonstrated longer aortic crossclamp and cardiopulmonary bypass times for patients who died. The prolonged exposure to cardiopulmonary bypass of patients undergoing reoperative AVR likely contributed to the increased overall mortality among these patients. The concurrent decrease in mortality associated with shorter cardiopulmonary bypass and aortic crossclamp times in the most recent era further suggests that prolonged pump and crossclamp times in the early operative eras grossly affected operative survival among patients undergoing reoperative AVR during that time.

There are important trends to note among patients undergoing reoperative AVR. We performed significantly more 
TABLE 5. Univariate analyses of preoperative risk factors and operative features for mortality in patients undergoing reoperative aortic valve replacement $(\mathbf{n}=\mathbf{1 9 1})$

\begin{tabular}{|c|c|c|c|}
\hline Variable & Survived $(\mathbf{n}=175)$ & Died $(n=16)$ & $P$ value \\
\hline \multicolumn{4}{|l|}{ Preoperative } \\
\hline Age at operation $(y$, mean $\pm S D)$ & $66.4 \pm 13.0$ & $67.6 \pm 15.1$ & .71 \\
\hline Male sex (no.) & $130(74.3 \%)$ & $10(62.5 \%)$ & .38 \\
\hline Peripheral vascular disease (no.) & $32(18.3 \%)$ & $1(6.3 \%)$ & .31 \\
\hline Cerebrovascular disease (no.) & $34(19.4 \%)$ & $3(18.8 \%)$ & $>.99$ \\
\hline Chronic lung disease (no.) & $22(12.6 \%)$ & $5(31.3 \%)$ & .06 \\
\hline Diabetes (no.) & $47(26.9 \%)$ & $3(18.8 \%)$ & .57 \\
\hline Dyslipidemia (no.) & $102(58.3 \%)$ & $6(37.5 \%)$ & .12 \\
\hline Coronary artery disease (no.) & $114(65.1 \%)$ & $6(37.5 \%)$ & .05 \\
\hline Hypertension (no.) & $107(61.1 \%)$ & $4(25.0 \%)$ & .01 \\
\hline \multicolumn{4}{|l|}{ New York Heart Association functional class (no.) } \\
\hline I & $11(6.3 \%)$ & $3(18.8 \%)$ & .10 \\
\hline II & $99(56.6 \%)$ & $5(31.3 \%)$ & .07 \\
\hline III & $57(32.6 \%)$ & $6(37.5 \%)$ & .78 \\
\hline IV & $8(4.6 \%)$ & $2(12.5 \%)$ & .20 \\
\hline Endocarditis (no.) & $13(7.4 \%)$ & $2(12.5 \%)$ & .36 \\
\hline Chronic renal insufficiency (no.) & $29(16.6 \%)$ & $3(18.8 \%)$ & .74 \\
\hline Ejection fraction $(\%$, mean $\pm \mathrm{SD})$ & $47.5 \pm 14.1$ & $48.3 \pm 10.4$ & .92 \\
\hline EuroSCORE (logistic, mean $\pm \mathrm{SD}$ ) & $22.8 \pm 15.4$ & $30.2 \pm 21.7$ & .05 \\
\hline EuroSCORE (standard, mean \pm SD) & $10.3 \pm 2.7$ & $11.2 \pm 3.4$ & .17 \\
\hline \multicolumn{4}{|l|}{ Operative } \\
\hline Isolated aortic valve replacement (no.) & $95(54.3 \%)$ & $9(56.3 \%)$ & $>.99$ \\
\hline Bioprosthetic valve (no.) & $117(66.9 \%)$ & $8(50.0 \%)$ & .18 \\
\hline Mechanical valve (no.) & $51(29.1 \%)$ & $4(25.0 \%)$ & $>.99$ \\
\hline Homograft (no.) & $2(1.1 \%)$ & $3(18.8 \%)$ & .004 \\
\hline \multicolumn{4}{|l|}{ Concomitant operations (no.) } \\
\hline Coronary artery bypass grafting & $53(30.3 \%)$ & $5(31.3 \%)$ & $>.99$ \\
\hline Aortic root operations & $8(4.6 \%)$ & $1(6.3 \%)$ & .55 \\
\hline Mitral valve procedures & $20(11.4 \%)$ & $2(12.5 \%)$ & $>.99$ \\
\hline Pulmonic valve procedures & $0(0.0 \%)$ & $0(0.0 \%)$ & NA \\
\hline Tricuspid valve procedures & $5(2.9 \%)$ & $0(0.0 \%)$ & $>.99$ \\
\hline Cardiopulmonary bypass time (min, mean $\pm \mathrm{SD}$ ) & $132.9 \pm 43.1$ & $209.5 \pm 89.4$ & .001 \\
\hline Aortic crossclamp time (min, mean $\pm \mathrm{SD}$ ) & $90.4 \pm 35.9$ & $145.3 \pm 73.3$ & .01 \\
\hline
\end{tabular}

NA, Not applicable.

reoperative AVRs in the most recent operative era than in eras A and B. Although New York Heart Association functional class IV was more common in the early operative era, patients in the most recent era were older and had higher preoperative EuroSCOREs. Consequently, these patients were sicker and carried higher preoperative risk than did those in the earlier eras. In addition, decreases in complications and mortality occurred in conjunction with an increase in the number of reoperative AVR cases at our institution. This is consistent with evidence that suggests that high-volume cardiac centers report improved operative outcomes. ${ }^{15}$ Otherwise, improvements in cardiac anesthesia, operative techniques (including less extensive dissection), and postoperative care likely played a fundamental role in our enhanced operative outcomes and decreased mortality over time. Recently, we demonstrated no differences in postoperative outcomes or mortality in reoperative cardiac surgery after
CABG with avoidance of clamping the internal thoracic artery. ${ }^{16}$ It is possible that this change in reoperative technique and minimal dissection may have contributed to the decreases in complication rates and mortality that we observed during the most recent operative era.

The effect of initial cardiac operation on reoperative AVR outcomes remains ill defined. Reoperative aortic valve surgery after previous $\mathrm{CABG}^{17}$ or $\mathrm{AVR}^{18}$ has in the past been associated with elevated risk of morbidity and mortality. Some studies, however, have recently demonstrated that previous CABG does not significantly increase mortality risk in reoperative AVR. ${ }^{19-21}$ These results are consistent with our observations that CABG and CABG with AVR as the initial operation did not significantly affect rates of postoperative complications or operative mortality relative to previous isolated AVR or other cardiac valve operations. 
Current treatment options for both primary and reoperative aortic valve surgery vary greatly. Options include aortic valve repair, traditional AVR with either biologic or mechanical prosthetic valves, pulmonary autograft (Ross procedure), and, most recently, transcatheter aortic valve implantation. In this study, insufficient numbers of aortic valve repair and Ross autograft procedures were performed to warrant useful statistical analyses. All AVRs performed among reoperative patients were open procedures with either bioprosthetic or mechanical valves. No percutaneous or transapical AVRs were performed during this study period. Early experience with percutaneous and transapical AVRs in high-risk patients with aortic valve disease have been mixed. Mortalities after percutaneous and transapical AVR have ranged from $7 \%$ to $14 \%$ in recent series. ${ }^{22-25}$ Relative to our reoperative AVR cohort, these mortality rates remain significantly elevated. Because the high-risk operative group in which percutaneous aortic valves are currently being used differs from reoperative AVR candidates, further investigation into the outcomes of these techniques will be required before advocating this technology as a primary approach to reoperative AVR.

Several paradoxic findings emerged during this study. We observed shorter aortic crossclamp times and lower rates of postoperative atrial fibrillation among patients undergoing reoperative AVR than among those undergoing primary AVR, as well as higher rates of coronary artery disease and hypertension among survivors of reoperative AVR survivors than among those who died. Unfortunately, we are unable to explain these results completely because of the admittedly small sample sizes of these study cohorts. We are thus constrained in our efforts to attribute any true clinical significance to these findings.

There are several limitations to this study. First, the retrospective nature of this study introduces inherent bias, and we are limited by variables that are collected in the STS database. Second, this study included a heterogeneous cohort. All patients undergoing an AVR were included during the referenced study period, regardless of concomitant cardiac operation. Because the numbers and types of concomitant operations varied among patients, small differences related to specific concomitant operations were difficult to assess. Third, the confounding effects of different operating surgeons over time were difficult to determine and may have influenced the trends observed in this study. Similarly, changes over time in both cardiac anesthesia and postoperative care are difficult to account for in data analyses. Finally, the total number of patients undergoing reoperative AVR was admittedly small relative to those undergoing primary AVR. Our ability to detect small differences between the groups was therefore limited, and our ability to define risk factors for reoperative AVR more clearly was constrained. Nevertheless, the observed trends in markedly improved postoperative complications and operative mortality among reoperative patients undergoing AVR during the recent era corroborates the important clinical contribution of this study to recently published data. In addition, our findings indicating improved results as our experience grew suggest that these high-risk procedures should be performed at high-volume centers.

\section{CONCLUSIONS}

In this study, reoperative AVR in the current era has been shown to carry similar morbidity and mortality to those of primary AVR. Risks of reoperation were unaffected by the initial cardiac operation. We therefore believe that reoperative AVR should be considered a safe operation for patients requiring surgical treatment for aortic valve disease after a previous sternotomy.

We thank Curtis Klann and Judy Smith for their assistance with the STS Database search and data collection.

\section{References}

1. Society of Thoracic Surgeons national cardiac surgical database. Chicago: Society of Thoracic Surgeons. c2008 [cited 2009]. Available at: http://www.sts.org/ sections/stsnationaldatabase/

2. Akins CW, Buckley MJ, Daggett WM, Hilgenberg AD, Vlahakes GJ, Torchiana DF, et al. Risk of reoperative valve replacement for failed mitral and aortic bioprostheses. Ann Thorac Surg. 1998;65:1545-52.

3. Biglioli P, Di Matteo S, Parolari A, Antona C, Arena V, Sala A. Reoperative cardiac valve surgery: a multivariable analysis of risk factors. Cardiovasc Surg. 1994;2:216-22.

4. Cohn LH, Aranki SF, Rizzo RJ, Adams DH, Cogswell KA, Kinchla NM, et al. Decrease in operative risk of reoperative valve surgery. Ann Thorac Surg. 1993;56:15-21.

5. Jones JM, O'Kane H, Gladstone DJ, Sarsam MA, Campalani G, MacGowan SW, et al. Repeat heart valve surgery: risk factors for operative mortality. $J$ Thorac Cardiovasc Surg. 2001;122:913-8.

6. Luciani N, Nasso G, Anselmi A, Glieca F, Gaudino M, Girola F, et al. Repeat valvular operations: bench optimization of conventional surgery. Ann Thorac Surg. 2006;81:1279-83.

7. Engoren M, Arslanian-Engoren C, Steckel D, Neihardt J, Fenn-Buderer N. Cost, outcome, and functional status in octogenarians and septuagenarians after cardiac surgery. Chest. 2002;122:1309-15.

8. Mehta RH, Eagle KA, Coombs LP, Peterson ED, Edwards FH, Pagani FD, et al. Influence of age on outcomes in patients undergoing mitral valve replacement. Ann Thorac Surg. 2002;74:1459-67.

9. Jamieson WR, Burr LH, Miyagishima RT, Janusz MT, Fradet GJ, Ling H, et al. Re-operation for bioprosthetic aortic structural failure-risk assessment. Eur $J$ Cardiothorac Surg. 2003;24:873-8.

10. Lytle BW, Cosgrove DM, Taylor PC, Gill CC, Goormastic M, Golding LR, et al. Reoperations for valve surgery: perioperative mortality and determinants of risk for 1,000 patients, 1958-1984. Ann Thorac Surg. 1986;42:632-43.

11. Piehler JM, Blackstone EH, Bailey KR, Sullivan ME, Pluth JR, Weiss NS, et al. Reoperation on prosthetic heart valves. Patient-specific estimates of in-hospital events. J Thorac Cardiovasc Surg. 1995;109:30-48.

12. Tyers GF, Jamieson WR, Munro AI, Germann E, Burr LH, Miyagishima RT, et al. Reoperation in biological and mechanical valve populations: fate of the reoperative patient. Ann Thorac Surg. 1995;60(2 Suppl):S464-9.

13. Vogt PR, Brunner-LaRocca H, Sidler P, Zund G, Truniger K, Lachat M, et al. Reoperative surgery for degenerated aortic bioprostheses: predictors for emergency surgery and reoperative mortality. Eur J Cardiothorac Surg. 2000;17:134-9.

14. Tabata M, Khalpey Z, Shekar PS, Cohn LH. Reoperative minimal access aortic valve surgery: minimal mediastinal dissection and minimal injury risk. $J$ Thorac Cardiovasc Surg. 2008;136:1564-8. 
15. Gammie JS, O'Brien SM, Griffith BP, Ferguson TB, Peterson ED. Influence of hospital procedural volume on care process and mortality for patients undergoing elective surgery for mitral regurgitation. Circulation. 2007;115:881-7.

16. Smith RL, Ellman PI, Thompson PW, Girotti ME, Mettler BA, Ailawadi G, et al. Do you need to clamp a patent left internal thoracic artery-left anterior descending graft in reoperative cardiac surgery? Ann Thorac Surg. 2009;87:742-7.

17. Odell JA, Mullany CJ, Schaff HV, Orszulak TA, Daly RC, Morris JJ. Aortic valve replacement after previous coronary artery bypass grafting. Ann Thorac Surg. 1996;62:1424-30.

18. Shemin RJ, Guadiani VA, Conkle DM, Morrow AG. Prosthetic aortic valves. Indications for and results of reoperation. Arch Surg. 1979;114:63-5.

19. Akins CW, Hilgenberg AD, Vlahakes GJ, Madsen JC, MacGillivray TE. Aortic valve replacement in patients with previous cardiac surgery. J Card Surg. 2004; 19:308-12.

20. Hirose H, Gill IS, Lytle BW. Redo-aortic valve replacement after previous bilateral internal thoracic artery bypass grafting. Ann Thorac Surg. 2004;78:782-5.

21. Sundt TM 3rd, Murphy SF, Barzilai B, Schuessler RB, Mendeloff EN, Huddleston $\mathrm{CB}$, et al. Previous coronary artery bypass grafting is not a risk factor for aortic valve replacement. Ann Thorac Surg. 1997;64:651-8.

22. Bleiziffer S, Ruge H, Mazzitelli D, Schreiber C, Hutter A, Laborde JC, et al. Results of percutaneous and transapical transcatheter aortic valve implantation performed by a surgical team. Eur J Cardiothorac Surg. 2009;35:615-21.

23. Tamburino C, Capodanno D, Mul EM, Scarabelli M, Cammalleri V, Barbanti M, et al. Procedural success and 30-day clinical outcomes after percutaneous aortic valve replacement using current third-generation self-expanding CoreValve prosthesis. I Invasive Cardiol. 2009;21:93-8.

24. Walther T, Falk V, Kempfert J, Borger MA, Fassl J, Chu MW, et al. Transapical minimally invasive aortic valve implantation; the initial 50 patients. Eur J Cardiothorac Surg. 2008;33:983-8.

25. Ye J, Cheung A, Lichtenstein SV, Pasupati S, Carere RG, Thompson CR, et al. Six-month outcome of transapical transcatheter aortic valve implantation in the initial seven patients. Eur J Cardiothorac Surg. 2007;31:16-21.

\section{Discussion}

Dr Leonard N. Girardi (New York, NY). I congratulate Dr LaPar for an excellent presentation and the University of Virginia group for outstanding results in a patient population that we are all seeing more of, those with previous cardiac surgery now requiring AVR. The $2 \%$ mortality in their last 100 cases is truly remarkable, actually lower than their mortality among patients undergoing primary AVR. To help us to learn more from their experience and perhaps incorporate some of their experiences and recommendations into our own practice, I have a few questions.

Dr LaPar, you were correct in assigning some of the credit for this improvement in mortality with time to improved surgical technique and a larger hospital experience with this patient population. The patients in the last era, however, about the last 100 patients, actually had better New York Heart Association functional class, had higher ejection fraction, underwent fewer urgent operations, and underwent fewer concomitant mitral valve and $\mathrm{CABG}$ procedures at the time of reoperative AVR. So, are we truly technically better than we were in a previous era, or are we just better at getting patients to the operating room earlier? That would include educating our cardiology counterparts in getting patients to the operating room earlier. Also, once we get them to the operating room, are we a little smarter not so much in how we do the operation technically but perhaps in what we don't do to the mitral valve and to the coronaries, to cut down on the increased crossclamp and bypass times that so significantly contributed to mortality in your series?

Dr LaPar. Dr Girardi, thank you for the feedback and those insightful questions. Certainly, patient selection is fundamental in improving operative outcomes and mortality. In this study, we believe that the trends that we had seen in declining operative mortality and outcomes are likely related to a combination of several factors. As you mentioned, we performed significantly more reoperative AVRs in the most recent era. We believe that as we do more surgery, we are getting better. In addition, the change in our surgical technique to include a minimal dissection of the heart has contributed to shorter aortic crossclamping and cardiopulmonary bypass times. We feel that these effects are significant in our results. We also, in the most recent times, have obtained more preoperative computed tomographic angiograms to help us to define vein graft anatomy better, as well as cardiac and mediastinal anatomy, and we believe that this may help in that patient selection and also in our operative planning. Finally, improvements in cardiac anesthesia and postoperative care with time have likely resulted in benefits for all cardiac operations.

With respect to preoperative risk, you mentioned ejection fraction and New York Heart Association functional class. We primarily thought to evaluate preoperative risk with EuroSCORE, and we did find higher EuroSCOREs in the most recent operative era. We consider higher EuroSCOREs to be representative of a sicker and older population in the most recent era. To rule out the effects of concomitant operations, we thought it was important to look at the isolated reoperative AVR group. We showed nearly identical trends and improved mortality within that group as well.

Dr Girardi. Second, and finally, if open reoperative AVR is to remain competitive with the less invasive approaches that we heard about earlier today, transapical and transfemoral AVR, especially in reoperations where they may actually have some obvious benefits, we have to continue to work on reducing the morbidity and mortality. The number one cause of mortality in your series was bleeding and post operative tamponade. Although in the last era you had a remarkable $5 \%$ reoperation or reexploration rate for bleeding, $43 \%$ of the deaths in this series were from tamponade and bleeding. So how are you handling that? Also, should we actually be a bit more aggressive and liberal about taking patients back for bleeding to avoid those catastrophic consequences of massive transfusion and unrecognized tamponade?

Dr LaPar. We could not agree more. At our institution, we perform early reexploration for all suspected cases of persistent postoperative bleeding or tamponade. Specifically, we perform early reexploration in the intensive care unit with standard operating room equipment, personnel, and procedure. Our philosophy is that early reexploration will prevent further postoperative complications and improve patient outcome. Furthermore, we believe that the higher postoperative bleeding rates of our earlier operative eras is related to more complex dissections in the past.

Dr Anthony L. Estrera (Houston, Tex). I congratulate you on a nice presentation. In doing reoperative cardiac surgery, we also apply this minimal dissection, or what I call directed dissection. More than half your cases had previous CABG. So my question is, how did you deal with the patent left internal thoracic artery?

Dr LaPar. Thank you for that question. One of the more recent philosophic changes in our operative techniques is that we do not routinely clamp the internal thoracic artery during reoperations. In fact, we recently published a series looking at this issue and found no difference in outcome or mortality with this technique. In adopting this approach, we have attained equal results while at the same time simplifying the operation and avoiding internal thoracic artery injury. 
Dr Sacha Salzberg (Zurich, Switzerland). Thank you for this nice presentation. I have a question regarding the technique of cannulation and how that has evolved with time. Have you used axillary cannulation? If so, how has that influenced outcomes and neurologic complications?

Dr LaPar. In this study, we did not specifically evaluate outcomes according to cannulation technique. We routinely use preop- erative computed tomographic angiography and intraoperative ultrasonography, however, to survey the ascending aorta to select an appropriate aortic cannulation site. Almost all the cases included in this study involved direct aortic cannulation. For cases in which aortic cannulation is difficult, we favor such approaches as axillary or innominate cannulation. 